\title{
The nonocclusive laser-assisted coronary anastomotic connector in an off-pump porcine bypass model
}

\author{
David Stecher, MD, ${ }^{a}$ Frebus J. van Slochteren, MSc, ${ }^{b}$ Imo E. Hoefer, MD, PhD ${ }^{c}$ \\ Gerard Pasterkamp, MD, PhD, ${ }^{\mathrm{c}}$ Cornelis A. F. Tulleken, MD, PhD, ${ }^{\mathrm{d}}$ Lex A. van Herwerden, MD, PhD, ${ }^{\mathrm{a}}$ \\ and Marc P. Buijsrogge, $\mathrm{MD}, \mathrm{PhD}^{\mathrm{a}}$
}

\begin{abstract}
Objectives: To facilitate minimally invasive coronary artery bypass grafting, a simplified alternative for handsutured anastomoses must be developed. We assessed the feasibility and anastomotic healing of the ameliorated Excimer laser-assisted nonocclusive anastomosis coronary prototype connector in an acute rabbit study (study 1) and in a long-term porcine off-pump coronary bypass study (study 2 ).
\end{abstract}

\begin{abstract}
Methods: Eighteen anastomoses were constructed on the abdominal aorta of the rabbit. In the porcine model, 15 left internal thoracic artery to left anterior descending coronary artery bypasses were evaluated intraoperatively and at 4 hours, 4 and 10 days, 2, 3, and 5 weeks, and 6 months (each $\mathrm{n}=2$ anastomoses). The anastomoses were examined by angiography, flow measurements, fractional flow reserve, coronary flow reserve, histologic features, and scanning electron microscopy.
\end{abstract}

Results: In study 1, all 18 anastomoses were patent and resisted supraphysiologic pressures $(\mathrm{n}=12,300 \mathrm{~mm}$ $\mathrm{Hg})$. In study 2 , the connector enabled nonocclusive and fast $(7.7 \pm 2.2$ minutes, mean \pm standard deviation) anastomosis construction. All but 1 of 15 anastomoses (owing to a technical error) were fully patent (FitzGibbon grade A) at follow-up. Histologic examination and scanning electron microscopy demonstrated complete endothelialization of the anastomoses at 10 days. At 6 months, no flow-limiting but streamline-covering intimal hyperplasia was shown (fractional flow reserve, $0.93 \pm 0.07$ mean \pm standard deviation).

Conclusions: The new nonocclusive coronary connector is easy to use, and the long-term results suggest favorable healing and remodeling in the porcine model. After downsizing, this anastomotic device, with its emphasis on zero ischemia and simplified prebounding of vessel walls, has intrinsic potential for minimally invasive off-pump coronary artery bypass surgery. (J Thorac Cardiovasc Surg 2014;147:1390-7)

Supplemental material is available online.

To facilitate the distal coronary anastomosis construction for minimally invasive coronary artery bypass grafting, we recently developed an Excimer laser-assisted nonocclusive anastomosis (ELANA) prototype coronary connector and assessed its feasibility in an acute rabbit abdominal aortic bypass model. ${ }^{1}$ As a result, we implemented several

From the Departments of Cardiothoracic Surgery, ${ }^{\mathrm{a}}$ Cardiology, ${ }^{\mathrm{b}}$ and Experimental Cardiology, ${ }^{\mathrm{c}}$ Heart and Lungs Division, and Department of Neurosurgery, ${ }^{\mathrm{d}}$ Brain Division, University Medical Center Utrecht, Utrecht, The Netherlands.

This study was supported by the University Medical Center Utrecht, ELANA b.v., and Eurostars EUREKA, project NewHeart (ESTAR10210).

Disclosures: Dr Tulleken holds shares in AMJ b.v., Utrecht, The Netherlands. Drs Stecher, Tulleken, and Buijsrogge are registered as co-inventors of patents regarding the ELANA technique, without financial benefits. All other authors have nothing to disclose with regard to commercial support.

Received for publication May 2, 2013; revisions received June 24, 2013; accepted for publication July 3, 2013; available ahead of print Aug 30, 2013.

Address for reprints: David Stecher, MD, Department of Cardiothoracic Surgery, University Medical Center Utrecht, Room E03.511, PO Box 85500, Utrecht 3508 GA The Netherlands (E-mail: dsteche2@umcutrecht.nl).

$0022-5223 / \$ 36.00$

Copyright $(2014$ by The American Association for Thoracic Surgery http://dx.doi.org/10.1016/j.jtcvs.2013.07.018 design modifications (ie, improving anastomotic hemostasis without mandatory sealing ${ }^{1}$ ).

In the present study, the initial safety and feasibility of the ameliorated ELANA coronary prototype connector were assessed in the same acute rabbit model (study 1). Subsequently, the connector was evaluated (ie, anastomotic healing and hemodynamic function) in an off-pump porcine left internal thoracic artery (LITA) to left anterior descending artery (LAD) survival study up to 6 months (study 2).

\section{METHODS \\ ELANA Coronary Connector and Procedure}

In contrast to the previous prototype, ${ }^{1}$ the novel ELANA connector (Figure 1, A) consists of a ring and a fork with 2 sharp pins. A spring (Peter Lazic, GmbH, Tuttlingen, Germany) at the back of the device enables the fork and ring of the connector to open and close with the use of an applicator (Aneurysm clip applier; Peter Lazic, GmbH). The connector (titanium; thickness, $0.25 \mathrm{~mm}$ ) is currently applicable to target vessels with an inner diameter of $\pm 2.4 \mathrm{~mm}(\approx 3.0 \mathrm{~mm}$ outer diameter [OD]; lumen, $4.5 \mathrm{~mm}^{2}$ ).

1. Mounting the graft. The graft (minimal OD of $2.0 \mathrm{~mm}$ ) is mounted onto the extravascular ring of the opened connector (arteriotomy, $\pm 3.0 \mathrm{~mm}$ long), using 4 to 8 Prolene 8-0 stitches (Ethicon, Somerville, NJ), such that the intima of the graft completely covered the inner surface of the ring (Figure 1,C). 


\section{Abbreviations and Acronyms \\ BENIS = blood-exposed nonintimal surface \\ CFR = coronary flow reserve \\ ELANA $=$ Excimer laser-assisted nonocclusive anastomosis \\ FFR = fractional flow reserve \\ $\mathrm{LAD}=$ left anterior descending artery \\ LITA $=$ left internal thoracic artery \\ OD $=$ outer diameter \\ $\mathrm{OPCAB}=$ off-pump coronary artery bypass}

2. Nonocclusive prebounding of graft to recipient artery. The mounted connector is opened by the applicator, and the fork is inserted into the recipient artery (Figure 1,D). Subsequently, the connector is closed by releasing the applicator (the connector stays in situ and is not removed). Hence, it is possible-owing to the nonocclusive prebounding of the graft onto the artery, before the arteriotomy-to check for proper positioning of the connector without a time constraint or blood actively obscuring the field. In the case of malposition, it is feasible to reopen the connector and reposition the device.

3. Laser-punched arteriotomy. Subsequently, a cone-shaped laser catheter ( $2.5 \mathrm{~mm}$ OD, laser fibers, $2.0 \mathrm{~mm}$ OD; Figure 1, $B$ ) is introduced intravascularly, by way of the distal-not blood exposed — end of the graft, into the connector. Vacuum suction ( 2 minutes) through the lumen of the laser catheter onto the recipient (eg, coronary) wall is initiated, the laser is activated, and the anastomosis is lasered open, resulting in an anastomotic orifice of \pm 1.8 to $2.0 \mathrm{~mm}$ in diameter $\left(\approx 2.5-3.1 \mathrm{~mm}^{2}\right.$, note: $\mathrm{LAD}$, $4.5 \mathrm{~mm}^{2}$ ). Subsequently, the laser catheter is retrieved, including the laser-punched fragment of the recipient wall (ie, "flap") by vacuum suction (the laser catheter does not weld or seal the anastomosis but "laser punches" an opening). Hence, an adventitial rim of the recipient wall, the laser edge, is exposed intraluminally (Figure $1, E) .{ }^{1}$ If the flap is not retrieved by the catheter (ie, an incompletely lasered flap is still
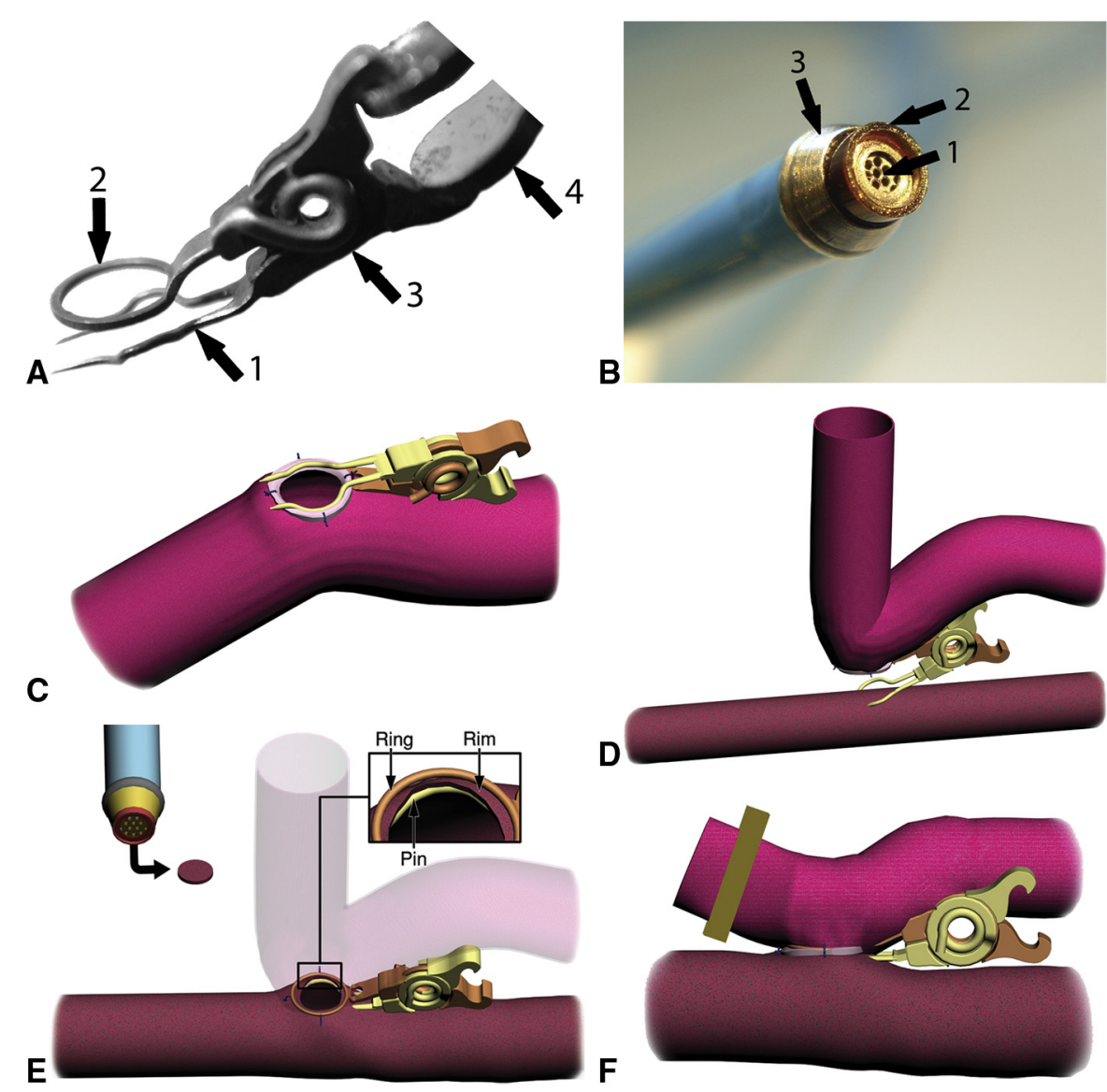

FIGURE 1. Excimer laser-assisted nonocclusive anastomosis coronary connector, laser catheter, and procedure. A, The slightly opened prototype connector with a fork $(1 ; 2.9 \mathrm{~mm}$ outer diameter [OD]). With 2 sharp pins ( $4.5 \mathrm{~mm}$ length) and a ring $(2 ; 3.4 \mathrm{~mm} \mathrm{OD})$. A spring (3) at the back of the device enables the ring and fork of the connector to open and close, with the use of an applicator (4). B, Laser catheter (2.5 mm OD): the grid of the vacuum channel (1) is located centrally and surrounded by laser fibers $(2 ; 2.0 \mathrm{~mm} \mathrm{OD})$. The cone-shaped tip (3; widest part, $3.0 \mathrm{~mm} \mathrm{OD})$ facilitates positioning and stabilization into the anastomotic connector. C, The graft is mounted onto the connector using stitches. The intima of the graft (highlighted) completely covers the inner surface of the ring of the connector. D, An applicator (not shown) is used to insert the connector. The pins of the connector puncture the recipient wall. E, After laser punching an opening into the anastomosis, the catheter-with a lasered fragment ("flap") of the recipient wall (graft illustrated transparently) — is retrieved. A small protruding adventitial rim of the recipient artery, the laser edge, is exposed intraluminally (see magnified subsection [complete intimal coverage of the ring of the connector by the graft not shown]). F, Final anastomosis, with a ligating hemoclip placed at the distal end of the graft. 
TABLE 1. Operative data

Anastomoses (n)

LITA $(\mathrm{mm}, \mathrm{OD})$

LAD (mm, OD)

Arteriotomy (mm; LITA)

Construction time* (min)

Mounting time (min)

Flap retrieval rate $(\%)$

Complete hemostasis $(\%)$

Extra stitch

Graft baseline flow $(\mathrm{mL} / \mathrm{min})$

Peak hyperemic flow response (peak/baseline flow) $\ddagger$

Data presented as mean minutes + standard deviation or \% (n). LITA, Left internal thoracic artery; $O D$, outer diameter; $L A D$, left anterior descending artery. *Included insertion, positioning laser catheter, vacuum suction, lasering, and closure of distal end of the graft. †In 1 of 15 anastomoses, the flap was not retrieved directly by the catheter, but the flap was removed manually. ‡After LITA clamping for 30 seconds, the coronary peak hyperemic flow response was determined as the mean peak graft flow divided by the mean baseline flow at $90 \mathrm{~mm} \mathrm{Hg}$.

partly attached to the recipient wall), the flap is retrieved manually (using micro-forceps) after opening the connector.

4. Bypass completion. After flap retrieval, the distal end of the graft is ligated using a hemoclip (Weck Hemoclip; Teleflex Medical, Research Triangle Park, NC; Figure 1, F).

\section{Initial Feasibility Study (Study 1) of the ELANA Coronary Connector in a Rabbit Model}

Eighteen anastomoses were constructed on the abdominal aorta (3.0 mm OD) of $3 \mathrm{New}$ Zealand white rabbits (female, $3 \mathrm{~kg}$; see Supplemental Methods section online) and evaluated intraoperatively and after death (follow-up period, 2.5 hours). ${ }^{1}$ All anastomotic procedures (Figure E1) were performed by 1 investigator (D.S.). The flap was directly retrieved in $16(89 \%)$ of the 18 anastomoses ( 2 were retrieved manually after opening the connector), and consistent graft flow was seen until termination $(26 \pm 8 \mathrm{~mL} / \mathrm{min}$ vs $27 \pm 8 \mathrm{~mL} / \mathrm{min}$ ). Hemostasis was established in $89 \%$ (16 of 18), and all anastomoses were patent. Histologic analysis $(n=6)$ showed reproducible and consistent constructions without detriment of the opposite and lateral walls of the recipient artery (Figure E2). Twelve ex vivo anastomoses were attached to a pressure system with water, and the pressure was increased by $100 \mathrm{~mm}$ $\mathrm{Hg} / \mathrm{min}$. All anastomoses $(\mathrm{n}=12)$ resisted supraphysiologic pressures (300 $\mathrm{mm} \mathrm{Hg}$ ).

This initial evaluation demonstrated the safety and feasibility of the new ELANA connector in a rabbit model. The improved features of the connector are as follows. First, the connector provides active compression of the graft onto the recipient artery by the spring at the back of the device, eliminating the use of a sealant, ${ }^{1}$ simplifying and accelerating anastomotic construction, and allowing resistance to supraphysiologic pressures. Second, cone shaping of the catheter ameliorates positioning and stabilization of the catheter into the connector and thus enhances flap retrieval ( $89 \%$ vs $83 \%$ in our previous study ${ }^{1}$ ). Third, by reopening the connector, a "bail out" (ie, manual flap retrieval) is offered in case the flap cannot be retrieved directly by the laser catheter.

\section{Evaluation of the ELANA Coronary Connector in the Off-Pump Porcine LITA-to-LAD Survival Study \\ (Study 2)}

Animals. Fifteen Dutch Landrace pigs (female; weight, 70-90 kg) were studied. The pigs were fed a normal diet and received humane care in compliance with the "Guide for the Care and Use of Laboratory Animals" prepared by the Institute of Laboratory Animal Resources, National Research Council (revised 1996). The animal experimentation committee of the Utrecht University approved the protocol. All pigs received $80 \mathrm{mg}$ of acetylsalicylic acid and $75 \mathrm{mg}$ clopidogrel orally daily, starting 3 days before surgery. This anticoagulation protocol was continued until termination (for details regarding the anesthesia and euthanasia protocol, see the Supplemental Methods section online).

\section{Surgery and Experimental Model}

After a partial sternotomy, LITA harvesting, and partial heparinization (activated clotting time $>200$; not counteracted at the end of the procedure), the ring of the connector was mounted onto the LITA (OD, 2.0-4.0 mm; Figure $1, C$ ), as previously described. Subsequently, after the LAD had been immobilized using the Octopus 3 Tissue Stabilizer (Medtronic, Minneapolis, Minn) at the intended site (OD, $3.0 \mathrm{~mm}$, measured using a caliper), the connector was inserted into the LAD with the pins directed downstream. After bypass construction, the LAD was ligated $\pm 1.0 \mathrm{~cm}$ upstream with 3 medium hemoclips. Finally, the LITA was tagged onto the epicardium, using Prolene 6-0, and the pericardium closed. Before chest closure, graft flow $(\mathrm{mL} / \mathrm{min})$ was monitored continuously for 90 minutes using a calibrated transit time flow probe (size 3S, flowmeter model T208; Transonic Systems, Ithaca, NY), at a mean blood pressure of $90 \mathrm{~mm} \mathrm{Hg}$.

The safety of the technique was assessed by anastomotic hemostasis, graft flow measurements, hemodynamic function (angiography, fractional flow reserve [FFR], and coronary flow reserve [CFR]), and anastomotic healing (ie, effects of implant and vessel wall compression by the connector; histologic examination and scanning electron microscopy). The anastomoses were evaluated intraoperatively $(\mathrm{n}=15)$ and at 4 hours, 4 and 10 days, 2, 3, and 5 weeks, and 6 months (each subgroup, 2 anastomoses) postoperatively. The follow-up was completed for 14 pigs (1 pig died postoperatively at \pm 12 hours).

\section{Hemostasis}

Hemostasis was quantified as completely hemostatic, oozing, or brisk leakage. Any leakage that was self-limiting or could be controlled by short tamponation (maximum, 3 minutes) was defined as oozing. If a stitch was necessary, the anastomosis required tamponation for more than 3 minutes, or the artery required temporary occlusion to obtain hemostasis, the leakage was defined as brisk.

\section{Angiography, FFR, and CFR}

All anastomoses $(\mathrm{n}=15)$, including in the 1 dead pig, were visualized at the follow-up examination using coronary angiography and graded by 2 observers according to the FitzGibbon criteria.

The FFR was calculated from 3 consecutive measurements directly distal (LAD) and proximal (LITA) to the anastomoses ( $\mathrm{n}=14$; at follow-up) and in the circumflex coronary artery (control coronary artery). The measurements were performed at baseline and during maximal hyperemia, induced by a bolus of intracoronary adenosine $(60 \mu \mathrm{g})$. The CFR was calculated as the ratio of maximal hyperemic flow velocity by the flow velocity at baseline (ComboWire XT, pressure/flow guide wire; Volcano Corp, Rancho Cordova, Calif).

\section{Histologic Analysis}

One anastomosis per subgroup ( $\mathrm{n}=8$; including 1 dead pig) was embedded in methyl methacrylate and sectioned in transverse planes (at $300-\mu \mathrm{m}$ intervals), starting $5 \mathrm{~mm}$ downstream and continuing to 5 mm upstream of the anastomosis, and stained with hematoxylin and eosin. ${ }^{1}$ The vessel wall apposition, protrusion of the intraluminal rim, anastomotic area, thrombus formation, intimal hyperplasia, blood-exposed nonintimal 


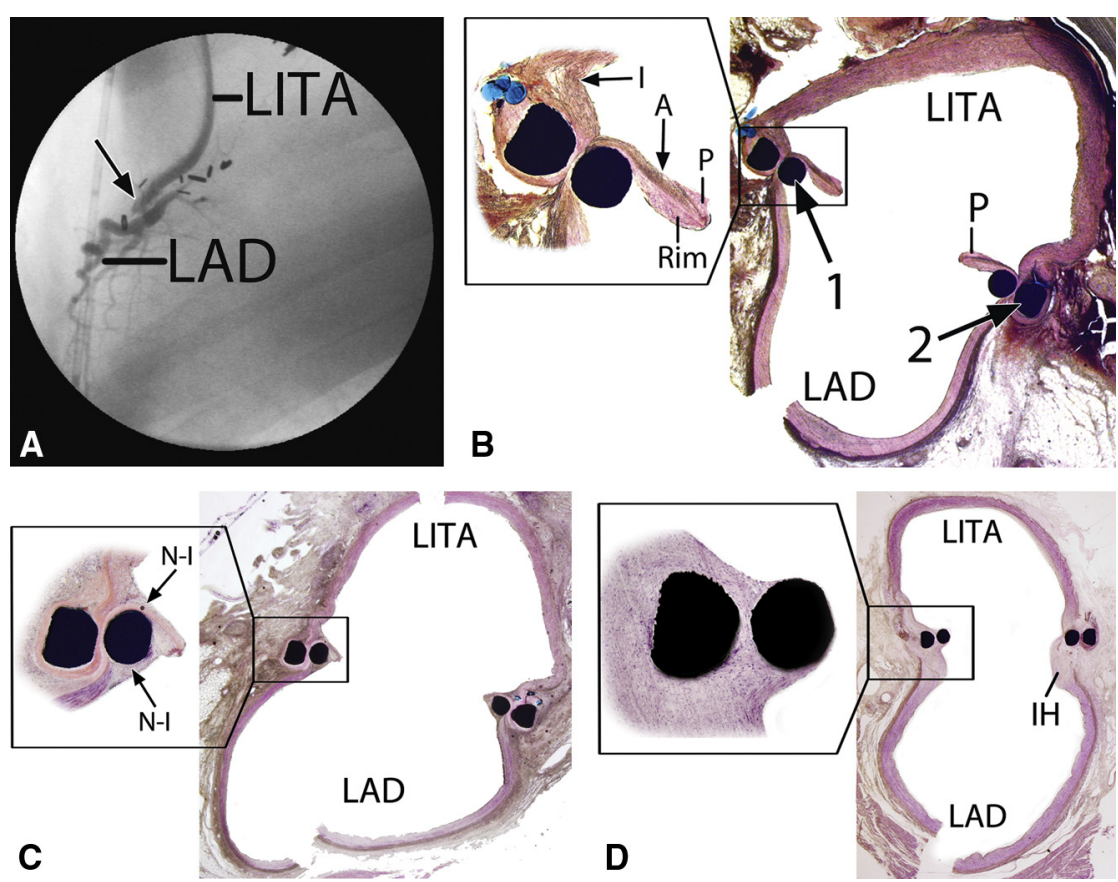

FIGURE 2. Six-month angiogram and histologic transverse sections of the Excimer laser-assisted nonocclusive anastomosis left internal thoracic artery (LITA)-to-left anterior descending artery ( $L A D)$ anastomosis at different points postoperatively. A, Angiogram of a LITA-to-LAD bypass at 6 months postoperatively. The arrow points to the anastomosis. Note, the relatively undersized anastomosis owing to the increased LAD (animal growth). B, At 4 hours, mid-anastomosis ( $12.5 \times$ magnification). A 0.2-mm compression surface of the LITA to LAD, between the fork (1) and the extravascular ring (2), is visible. The magnified $(40 \times)$ subsection demonstrates the laser rim with a sharp laser-cut edge $($ Rim $)$, with limited platelets and fibrin coverage $(P)$, and the intima (I)-adventitia (A) vessel wall apposition. C, At 10 days, mid-anastomosis $(12.5 \times)$. The laser rim is retracting, and neointima formation $(N-I)$ covers the adventitial rim and the intraluminally exposed fork (subsection, $40 \times$ ). D, At 6 months, mid-anastomosis $(12.5 \times)$. Streamlining intimal hyperplasia $(I H)$ is seen. Note, the remodeled laser rim and viable intima in between the fork and ring (ie, the compression surface), without medial necrosis. No chronic inflammatory cell reaction is seen (subsection, 100×). A small layer of endothelial cells covers the intraluminal fork of the connector. Note, interruption of the inferior coronary wall caused by longitudinally opening of the coronary artery before inspection.

surface $^{2}$ (BENIS; intraluminal exposure of the medial, adventitial [intraluminal exposed rim], and connector surface), acute and chronic inflammatory cell reaction (polymorphonuclear cells, macrophages, and foreign body giant cells), and tissue damage were recorded and assessed, as previously described. ${ }^{1}$ The measurements were performed using the software package AnalySiS (Soft-Imaging Software GmbH, Münster, Germany).

\section{Scanning Electron Microscopy}

The intravascular anastomotic surface of 1 anastomosis per subgroup $(\mathrm{n}=7)$ was evaluated (ie, assessment of endothelial and/or thrombocyte coverage) using a scanning electron microscope (Philips XL30LAB; FEI Europe, Eindhoven, The Netherlands; see the Supplemental Methods online).

\section{Statistical Analysis}

The data are presented as the mean \pm standard deviation or as noted otherwise.

\section{RESULTS \\ Surgery}

All anastomotic procedures were performed by 1 investigator (D.S.; operative data listed in Table 1). After grafting, all anastomoses $(\mathrm{n}=15)$ demonstrated consistent graft flow $(38 \pm 14 \mathrm{~mL} / \mathrm{min}$ at time $=0 ; 36 \pm 10 \mathrm{~mL} / \mathrm{min}$ at time $=90$ minutes). Of all 15 anastomoses, 1 oozed and 1 had brisk leakage. However, after repositioning of the latter

TABLE 2. Postoperative and postmortem measurements

\begin{tabular}{lcccccc}
\hline Interval & Lumen area* $\left(\mathbf{m m}^{\mathbf{2}}\right)$ & Anastomotic orifice $\dagger\left(\mathbf{m m}^{\mathbf{2}}\right)$ & LITA-LAD FFR & CFR & Cx FFR & CFR \\
\hline $4 \mathrm{~h}$ & $4.5 \pm 1.4$ & $2.6 \pm 0.3$ & $0.82 \pm 0.17$ & $3.0 \pm 0.2$ & $0.96 \pm 0.01$ & $4.7 \pm 0.6$ \\
$35 \mathrm{~d}$ & $6.4 \pm 1.0$ & $2.9 \pm 0.2$ & $0.95 \pm 0.02$ & $2.8 \pm 0.1$ & $0.98 \pm 0.01$ & $2.4 \pm 1.0$ \\
$180 \mathrm{~d}$ & $8.7 \pm 0.3$ & $3.7 \pm 0.5$ & $0.93 \pm 0.07$ & $4.6 \pm 0.7$ & $0.99 \pm 0.01$ & $3.5 \pm 0.1$ \\
\hline
\end{tabular}

Data presented as mean \pm standard deviation. $F F R$, Fractional flow reserve; $C F R$, coronary flow reserve; $C x$, circumflex coronary artery (control); LITA, left internal thoracic artery; $L A D$, left anterior descending artery. *Lumen area was determined using angiography $(\mathrm{n}=2)$ of reference $\mathrm{LAD}(1.0 \mathrm{~cm}$ downstream to the anastomosis $) . \dagger$ Anastomotic orifice determined by histologic examination $(\mathrm{n}=1)$ and scanning electron microscopy $(\mathrm{n}=1)$; note, the anastomotic orifice increased over time and the LAD increased because of growth of the animal. 

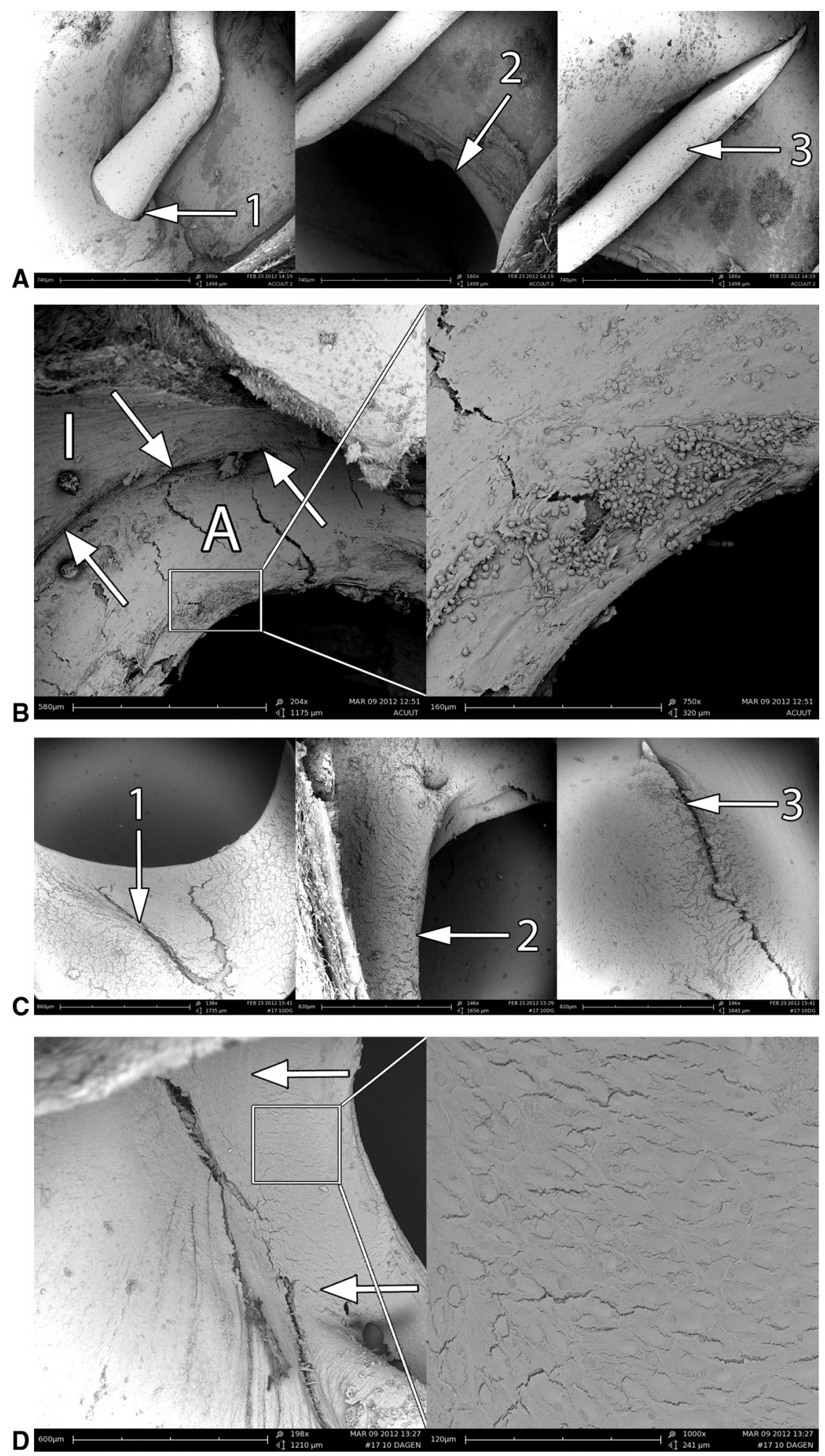

FIGURE 3. Scanning electron microscopy of the Excimer laser-assisted nonocclusive anastomosis left internal thoracic artery (LITA)-to-left anterior descending artery $(L A D)$ anastomosis at different points postoperatively. A and C, View from inside the LAD. From left to right: heel, middle, and toe of the anastomosis. B and D, View from inside the LITA. A, At 4 hours (160× magnification). The puncture site of the fork through the coronary wall (1), laser rim (2), and pin of the fork (3). No endothelial damage and limited platelets and fibrin coverage of the fork are seen. B, At 4 hours (204×). The intima (I)-adventitia $(A)$ vessel wall apposition (arrows). The magnified $(750 \times)$ subsection demonstrates the adventitial laser rim with limited fibrin and platelets coverage. Note, the sharp laser cut through the coronary artery. C and D, View at 10 days (from left to right, $138 \times, 146 \times$, and $146 \times$ ). Complete endothelialization is 
(ie, connector inserted slightly more distal into the LAD), complete hemostasis was obtained. None of the pigs showed ventricular arrhythmias or died during the operative procedure. The activated clotting time at 0 minutes, during the anastomotic procedure, and at 140 minutes after heparinization was $100 \pm 9,258 \pm 89$, and $145 \pm 41$ seconds, respectively.

\section{Follow-up}

The scheduled follow-up examination was completed for 14 pigs. All anastomoses were patent at follow-up, except for 1, which was occluded (assessed by postmortem angiography and histologic examination; the pig had been found dead at \pm 12 hours postoperatively). A technical error of the laser during anastomotic construction resulted in an asymmetric intraluminally exposed adventitial rim (left, 0.54; right, $0.36 \mathrm{~mm}$ ). Subsequent postmortem histologic examination showed a fresh anastomotic-occluding thrombus attached to the rim.

\section{Angiography, FFR, and CFR Measurements}

All but 1 of 15 anastomoses were fully patent at follow-up (FitzGibbon grade A; Figure 2, A). The mean FFR of the anastomoses at the 4-hour follow-up examination was 0.82 \pm 0.17 and increased over time to $0.93 \pm 0.07$ at 6 months. The mean CFR increased over time to $4.6 \pm 0.7$ at 6 months. The mean FFR and CFR in the control circumflex coronary artery at 4 hours was $0.96 \pm 0.01$ and $4.7 \pm 0.6$ and at 6 months was $0.99 \pm 0.01$ and $3.5 \pm 0.1$, respectively (Table 2).

\section{Histologic Findings}

Anastomotic healing and remodeling. In all anastomoses, the intima of the LITA was opposed to the adventitia of the LAD. A protruding rim of the latter (the laser rim; mean length, $0.63 \pm 0.09 \mathrm{~mm}$ and $0.42 \pm 0.04$ at 4 hours and 4 days, respectively), with a sharp laser-cut edge and limited platelets and fibrin coverage, was observed at 4 hours (Figure 2, $B$ ) and 4 days. The pins of the fork, distal to the anastomotic orifice, were compressed onto the upper wall of the LAD, without detriment.

At 10 (Figure 2, C) and 14 days, neointima formation covered both the retracted adventitial rim of the LAD and the intraluminally exposed fork of the connector. At 3 and 5 weeks, intimal hyperplasia had completely covered the anastomotic line, without narrowing the lumen.

At 6 months, the spring of the connector was fully integrated between the LITA and the LAD without erosion damage, and the coronary adventitial laser rim had been remodeled and replaced by neointimal tissue (Figure 2, $D$ ). In addition, the pins of the connector at the toe of the anastomosis were completely integrated into the upper wall of the LAD (Figure E3). At the mid of the anastomosis, the fork of the connector was covered by a thin layer of endothelial cells. The anastomotic recesses at both sides of the connector (ie, in- and outflow) were filled with streamlining intimal hyperplasia, which was, however, less abundant than that seen at 5 weeks, suggesting at least stabilization of the neointima formation. The anastomotic orifice area had expanded over time from $2.6 \pm 0.3 \mathrm{~mm}^{2}$ at 4 hours (LAD, $4.5 \pm 1.4 \mathrm{~mm}^{2}$ ) to $3.7 \pm 0.5 \mathrm{~mm}^{2}$ at 6 months (LAD, $8.7 \pm 0.3 \mathrm{~mm}^{2}$; Table 2).

BENIS area. The anastomosis showed a total BENIS area of $7.9 \mathrm{~mm}^{2}$ (intraluminal connector surface area, $\sim 3.8 \mathrm{~mm}^{2}$; and intraluminal exposed rim, $\sim 4.1 \mathrm{~mm}^{2}$ ) at 4 hours. The BENIS area gradually reduced to 0 over time (at 10 days, the nonintimal surface was fully covered with neointima). Medial necrosis, tissue damage, and inflammation. Compression of both vessel walls, between the ring and fork, was observed with some pyknosis (ie, degeneration) of smooth muscle cell nuclei at 4 hours and 4 days. However, no medial necrosis was seen at the compression surface, and no adverse remodeling (eg, erosion, luxation, or pseudoaneurysm formation) was demonstrated over time. The arterial walls of the LAD and LITA were unaffected, without thermal damage due to the laser, and without acute or long-term inflammation over time.

\section{Scanning Electron Microscopy}

After 4 hours, a clear demarcation between the endothelial surface of the coronary artery and the connector (Figure 3,A) and between the endothelium of the LITA and the adventitial laser rim (Figure 3,B) was observed. No endothelial damage of the coronary artery or of the graft (eg, due to laser damage) was seen; however, limited platelets and fibrin coverage of the connector and rim were seen. Additionally, a sharp laser cut through the coronary artery was demonstrated.

At 4 days, partial coverage of the connector and rim with platelets, fibrin, and endothelial cells was seen, demonstrating initiation of endothelialization of the nonintimal surface that was completed after 10 days (Figure 3, $C$ and $D$ ). A continuous endothelial surface from the coronary artery over the connector and the rim into the LITA (Figure 3, D, 1000× magnified subsection) could be seen at 10 days.

At 14, 21, and 35 days and 6 months, no differences in the anastomotic surface were found compared with the anastomosis at 10 days. All anastomoses showed patent

demonstrated. Note, the continuity of the endothelial surface from the lumen of the coronary artery over the connector $(\mathrm{C} ; 1$, heel; 2 , middle; 3 , toe $)$ and adventitial rim, to the LITA (D; $198 \times)$. Arrows point to the former intima $(I)$-adventitia $(A)$ vessel wall apposition border, and the magnified $(1000 \times)$ subsection shows a continuum of endothelium covering the rim. 
anastomotic orifices, that had expanded over time, completely covered with endothelial cells (Table 2).

\section{DISCUSSION}

After initial testing in the acute rabbit bypass model, the main purpose of the porcine off-pump coronary artery bypass (OPCAB) survival study was to investigate the healing with the novel ELANA coronary connector (ie, BENIS, the residual implant, and the active compression of the vessel walls). Favorable healing and remodeling were demonstrated despite the inherent undersize of the anastomosis, with sufficient hemodynamic function over time.

\section{Healing and Remodeling of the Alternative Vessel Wall Connection}

Despite the unconventional construction (ie, apposition of the intima of the graft to the adventitia of the coronary artery) and the undersized anastomotic geometry, favorable anastomotic healing and remodeling were demonstrated. At 10 days, the nonintimal surface was completely covered with neointima (Figures 2 and 3). In addition, the anastomotic orifice tended to expand over time by retraction of the laser rim, and no flowlimiting (FFR, $0.93 \pm 0.07$ ) intimal hyperplasia was seen at 6 months in the porcine model (with the latter follow-up duration comparable to 1.5-3 years of followup of the stented human coronary artery ${ }^{3}$ ). Supported by earlier experiments with laser-assisted anastomosis constructions in rabbits ${ }^{1,4}$ and pigs, ${ }^{5}$ no endothelial damage or thermal effects of the laser were found in our experimental coronary artery bypass grafting study. Moreover, 1 of the concerns with the use of a laser and a compression device is the risk of pseudoaneurysm formation. ${ }^{6}$ However, at 6 months, no adverse remodeling or anastomotic aneurysm formation and no erosion effects of the implant were observed.

\section{Facilitating Minimally Invasive Distal Coronary Anastomosis Construction}

The connector includes several interesting features, compared with other coronary anastomotic connectors, ${ }^{2,7-10}$ to facilitate minimally invasive or endoscopic distal coronary anastomosis construction. First, the most discriminating feature is the relatively simple first step of nonocclusive prebounding of the vessel walls, which enables anastomotic construction in a bloodless field, without a time constraint. Hence, coronary conditioning (ie, shunting or snaring ${ }^{7-10}$ ) would be redundant. Similarly, neither placing additional stitches to obtain hemostasis ${ }^{8}$ nor a separate incision into the target coronary would be necessary, ${ }^{7-10}$ reducing manipulation and conditioning of the coronary artery. Most importantly, this will simplify the off-pump bypass procedure, especially in an endoscopic or minimally invasive setting. Moreover, owing to the 2-step approach, it is feasible, in the case of malpositioning, before to the point of no return (ie, the arteriotomy), to reopen and reposition the connector.

Second, the ELANA connector is a low-profile device, without a bulky device-deployment system ${ }^{7-10}$; thus, it will not hamper the bypass construction on difficult-toreach or remote areas of the heart.

\section{Limitations of the ELANA Coronary Connector}

The connector requires optimization steps before clinical introduction. The round and fixed configuration of the anastomosis combined with the use of a cone-shaped laser catheter resulted, at least initially, in a relative undersizing of the anastomosis (Table 2; FFR, $0.82 \pm 0.17$ ) by extension of the blood-exposed adventitial laser rim (Figure 2, $B$; rim length, $0.63 \pm 0.09 \mathrm{~mm}$ vs $0.11 \pm 0.12 \mathrm{~mm}$ in a previous study ${ }^{1}$ without a cone-shaped catheter), and thus applied to more than half of the total BENIS. The BENIS, however, is inherent to distal anastomotic connectors. ${ }^{2,7-10}$ Buijsrogge et $\mathrm{al}^{11}$ stated that BENIS intima-adventitia apposition per se is not detrimental in a porcine low-flow LITA-to-LAD bypass model; however, the tolerance to additional technical errors becomes smaller. In the present study, the additional technical error of an asymmetrically lasered rim resulted in a fatal thrombotic occlusion in 1 pig. Thus, modifications to both the laser catheter and the anastomotic configuration (ie, oval shaped) will result in a relatively larger anastomotic orifice (proper matching of the anastomotic area to the target coronary artery area), a reduction in the nonintimal surface within the anastomosis, and optimal flap retrieval.

\section{Future Perspectives}

A new downsized, oval-shaped ELANA connector (anastomotic orifice, $\approx 1.3 \mathrm{~mm}^{2}$ ) is being designed. It can be mounted completely suturelessly and will be applicable to target coronary arteries with an OD of $1.5 \mathrm{~mm}$ (ie, \pm 1.2 $\mathrm{mm}$ inner diameter; $\approx 1.1 \mathrm{~mm}^{2}$ ). Before clinical application in coronary artery bypass grafting, the safety and quality of this downsized connector will be evaluated in both a long-term experimental porcine OPCAB study and a human atherosclerotic model.

\section{CONCLUSIONS}

The new nonocclusive coronary connector is easy to use, and the long-term results suggest favorable healing and remodeling in the porcine OPCAB model. After downsizing, this coronary anastomotic device, with its emphasis on zero ischemia and simplified prebounding of vessel walls, has intrinsic potential for minimally invasive or endoscopic OPCAB surgery. 
We acknowledge the constructive contributions of Glenn Bronkers, Cees Verlaan, Rik Mansvelt Beck, Niklank Noest, Noortje van den Dungen, Marlijn Jansen, Joyce Visser, Merel Schurink, Sander van Thoor, Saskia Redegeld, André van Dieren, Aryan Vink, Steven Chamuleau, Tycho van der Spoel, Sanne Jansen of Lorkeers, Petra van der Kraak, Chris Schneijdenberg, and colleagues from the Utrecht University Central Animal Facilities.

\section{References}

1. Stecher D, de Boer B, Tulleken CA, Pasterkamp G, van Herwerden LA, Buijsrogge MP. A new nonocclusive laser-assisted coronary anastomotic connector in a rabbit model. J Thorac Cardiovasc Surg. 2013;145:1124-9.

2. Scheltes JS, van Andel CJ, Pistecky PV, Borst C. Coronary anastomotic devices: blood-exposed non-intimal surface and coronary wall stress. J Thorac Cardiovasc Surg. 2003;126:191-9.

3. Fishell TA, Virmani R. Intracoronary brachytherapy in the porcine model: a different animal. Circulation. 2001;104:2388-90.

4. Tulleken CA, Verdaasdonk RM, Berendsen W, Mali WP. Use of the Excimer laser in high-flow bypass surgery of the brain. $J$ Neurosurg. 1993;78:477-80.
5. Streefkerk HJ, Bremmer JP, Tulleken CA. The ELANA technique: high flow revascularization of the brain. Acta Neurochir Suppl. 2005;94:143-8.

6. Grubbs PE, Wang S, Marini C, Rose DM, Cunningham JN. Enhancement of $\mathrm{CO}_{2}$ laser microvascular anastomoses by fibrin glue. J Surg Res. 1988;45: 112-9.

7. Suyker WJ, Buijsrogge MP, Suyker PT, Verlaan CW, Borst C, Grundeman PF Stapled coronary anastomosis with minimal intraluminal artifact: the S2 Anastomotic System in the off-pump porcine model. J Thorac Cardiovasc Surg. 2004;127:498-503.

8. Matschke KE, Gummert JF, Demertzis S, Kappert U, Anssar MB, Siclari F, et al. The Cardica C-Port System: clinical and angiographic evaluation of a new device for automated, compliant distal anastomoses in coronary artery bypass grafting surgery-a multicenter prospective clinical trial. J Thorac Cardiovasc Surg. 2005;130:1645-52.

9. Carrel T, Englberger L, Keller D, Windecker S, Meier B, Eckstein F. Clinical and angiographic results after mechanical connection for distal anastomosis in coronary surgery. J Thorac Cardiovasc Surg. 2004;127:1632-40.

10. Filsoufi F, Farivar RS, Aklog L, Anderson CA, Chen RH, Lichtenstein S, et al. Automated distal coronary bypass with a novel magnetic coupler (MVP system). J Thorac Cardiovasc Surg. 2004;127:185-92.

11. Buijsrogge MP, Grundeman PF, Verlaan CW, Borst C. Unconventional vessel wall apposition in off-pump porcine coronary artery bypass grafting: low versus high graft flow. J Thorac Cardiovasc Surg. 2002;123:341-7. 


\section{SUPPLEMENTAL METHODS \\ Animals}

Rabbits. Three female New Zealand white rabbits (weight, $3 \mathrm{~kg}$ ) were used. The rabbits were fed a normal diet and received humane care in compliance with the "Guide for the Care and Use of Laboratory Animals" (Institute of Laboratory Animal Resources, National Research Council [revised 1996]). The animal experimentation committee of the Utrecht University approved the protocol. The rabbits did not receive any anticoagulant or antiplatelet medication preoperatively.

Anesthesia was induced with acepromazine $(0.5 \mathrm{mg} / \mathrm{kg})$, methadone $(1 \mathrm{mg} / \mathrm{kg})$ intramuscularly and etomidate $(0.3$ $\mathrm{mg} / \mathrm{kg}$ ) intravenously. After intubation and ventilation, anesthesia was maintained by continuous intravenous infusion of midazolam $(50 \mu \mathrm{g} / \mathrm{hr} / \mathrm{kg})$ and sufentanil $(50 \mu \mathrm{g} / \mathrm{hr} /$ $\mathrm{kg}$ ). The rabbit was placed in a supine position, and the mean arterial blood pressure was maintained at $60 \mathrm{~mm}$ $\mathrm{Hg}$ for the whole procedure. The rabbits were killed with $200 \mathrm{mg} / \mathrm{kg}$ sodium pentobarbital intravenously.

Pigs. Anesthesia was induced with ketamine $(10 \mathrm{mg} / \mathrm{kg})$, midazolam $(0.5 \mathrm{mg} / \mathrm{kg})$, and atropine $(0.04 \mathrm{mg} / \mathrm{kg})$ intramuscularly. Each pig received antibiotic prophylaxis (Augmentin 1000/100 mg), thiopental sodium (4 mg/kg), midazolam $(0.5 \mathrm{mg} / \mathrm{kg})$, and sufentanil citrate $(6 \mu \mathrm{g} / \mathrm{kg})$ through an intravenous line. The pigs were intubated and ventilated with a mixture of oxygen and air (1:1 volume/ volume). Anesthesia was maintained by a continuous intravenous infusion of midazolam $(0.7 \mathrm{mg} / \mathrm{kg} / \mathrm{hr})$, analgesia was obtained with an infusion of sufentanil citrate (6 $\mu \mathrm{g} / \mathrm{kg} / \mathrm{hr}$ ), and muscle relaxation with pancuronium

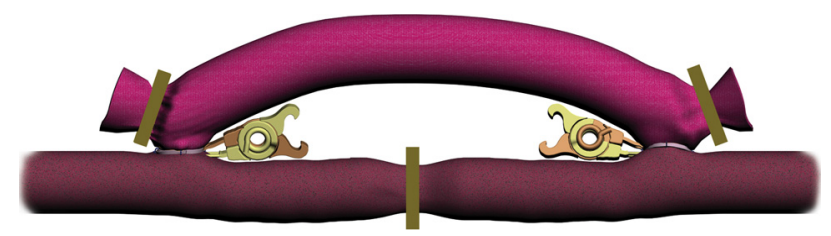

FIGURE E1. The rabbit abdominal aortic Excimer laser-assisted nonocclusive anastomosis bypass model. Final abdominal aortic bypass with 2 anastomoses. Ligating hemoclips are placed at both ends of the graft, and a hemoclip occludes the abdominal aorta in between the 2 anastomoses. bromide $(0.1 \mathrm{mg} / \mathrm{kg} / \mathrm{hr})$. During the operation, each pig received a continuous infusion of saline solution (300 $\mathrm{mL} / \mathrm{hr}$ ). Metoprolol was administered intravenously (range, $5-20 \mathrm{mg}$ ) to reduce the mechanical irritability of the heart until a heart rate of approximately 70 beats/ min was obtained. Postoperatively, synulox $(150 \mathrm{mg} / 20$ $\mathrm{kg}$ ) was administered, and analgesia was obtained with durogesic $25 \mu \mathrm{g}$ transdermally for 3 days. The pigs were killed with sodium pentobarbital $(200 \mathrm{mg} / \mathrm{kg})$ intravenously, after having been heparinized to obtain an activated clotting time (Hemotec, Englewood, Colo) of at least 4 times the control value.

\section{Scanning Electron Microscopy}

In the porcine model, 1 anastomosis per subgroup $(\mathrm{n}=7)$ was fixated in $2 \%$ glutaraldehyde solution buffered in 0.1 $\mathrm{M}$ purified phosphate buffer. Fixation was completed after the anastomosis was left in $1 \%$ buffered osmium tetroxide for 1 hour. After fixation, the anastomosis was dehydrated in a graded series $(50 \%, 70 \%, 90 \%$, and $100 \%)$ of ethanol and dried in liquid carbon dioxide using the critical point method. Subsequently, the back wall of the left anterior descending artery (LAD) and the upper wall of the left internal thoracic artery (LITA) at the anastomotic site were opened longitudinally, fixed on scan tubs, and covered with a thin layer of platinum by sputter processing to enhance the image quality.

The intravascular anastomotic surface was evaluated (ie, assessment of endothelial and/or thrombocyte coverage) using a scanning electron microscope (Philips XL30LAB; FEI Europe, Eindhoven, The Netherlands).

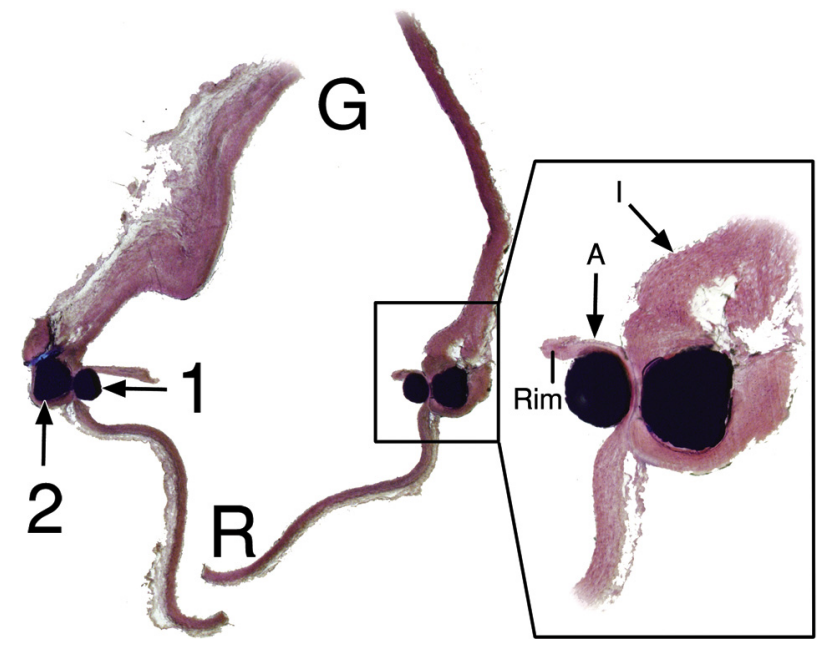

FIGURE E2. Histologic section of the Excimer laser-assisted nonocclusive anastomosis in the rabbit abdominal aortic bypass model (transverse section; $12.5 \times$ magnification). The fork (1) and ring (2) of the anastomotic connector are visible. The magnified $(40 \times)$ subsection demonstrates the intima $(I)$-adventitia $(A)$ vessel wall apposition and the sharp laser-cut edge (Rim). Compression of the recipient vessel wall between the ring and fork is seen. $G$, Lumen of the graft; $R$, recipient artery. 


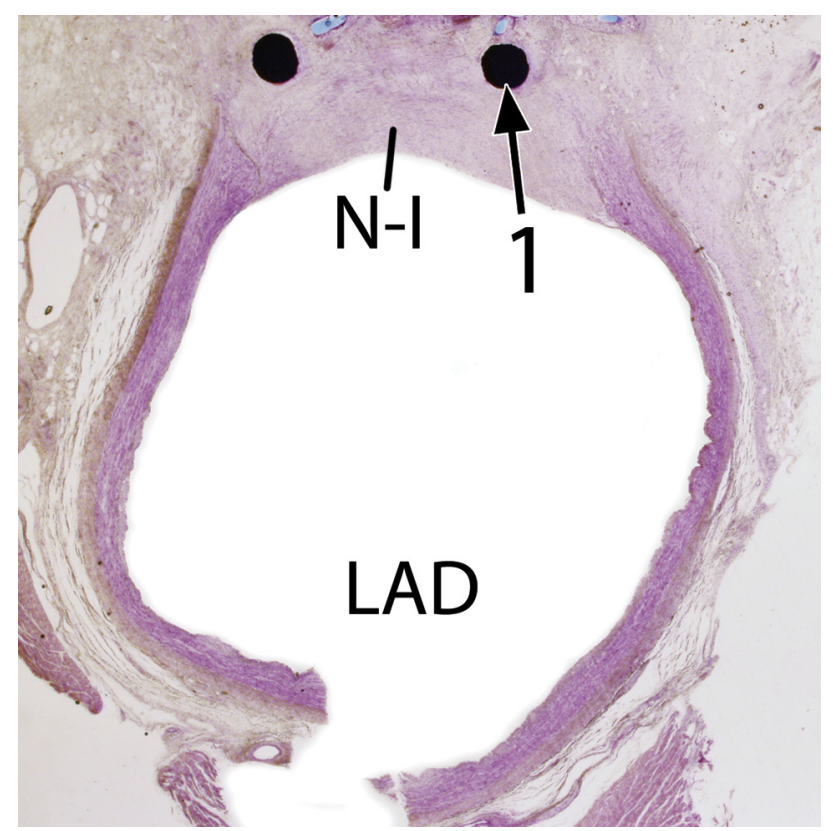

FIGURE E3. Histologic section of the left internal thoracic artery (LITA)to-left anterior descending artery $(L A D)$ Excimer laser-assisted nonocclusive anastomosis at 6 months in the porcine model (transverse section; $12.5 \times$ magnification). The pins of the connector (1) at the toe of the anastomosis (ie, distal to the anastomosis) are integrated into the upper wall of the LAD. $N-I$, Neointima formation. 